\title{
The Small-Péclet-Number Approximation for Radiative Zones and its Application to Shear Layer Instabilities
}

\author{
F. Lignières \\ Laboratoire d'Astrophysique de Toulouse et Tarbes, UMR CNRS 5572, \\ Observatoire Midi-Pyrénées, 31400 Toulouse, France
}

\begin{abstract}
Thermal diffusivities within stars are higher by several orders of magnitude than in the earth atmosphere. This particularity of the stellar fluid has subtle effects on the dynamics of stably stratified radiative zones as it contributes to favour some flows and supress others. An asymptotic analysis of hydrodynamic equations for large values of the thermal diffusivity is presented and this results in a simplification of the dynamical effects of the thermal diffusivity. This asymptotic behavior proved to be very useful to understand shear instabilities of Kelvin-Helmholtz type.
\end{abstract}

Understanding flow dynamics within stellar radiative zones constitutes a major challenge for the current theory of stellar evolution. We consider here the dynamical effects of the very high thermal diffusivity of radiative origin. This property of the stellar fluid is expected to strongly affect the flow dynamics when the time scale of thermal diffusion $t_{\kappa}$ is shorter than the dynamical time scale $t_{D}$, that is when the Péclet number $P_{e}=t_{\kappa} / t_{D}$ is smaller than one.

If we assume that, in the limit of small Péclet number, the velocity field $\mathbf{u}$ and the temperature deviation from the initial temperature profile, $\theta$, behave like Taylor series of $P e$, then the solutions of the Boussinesq equations are identical to the solutions of the following system up to the first order in $P_{e}$. We called this asymptotic limit the small-Péclet-number approximation:

$$
\begin{cases}\frac{\partial \mathbf{u}}{\partial t}+\mathbf{u} \cdot \nabla \mathbf{u} & =-\nabla p+R \psi \mathbf{e}_{z}+\frac{1}{R_{e}} \nabla^{2} \mathbf{u} \\ w & =\nabla^{2} \psi \\ \nabla \cdot \mathbf{u} & =0\end{cases}
$$

where $\psi=\theta / P_{e}$. It must be noted that, instead of two distinct non-dimensional number, the Richardson number $R_{i}$ and the Péclet number $P_{e}$, these equations depends on a unique non-dimensional number, namely $R=R_{i} P_{e}$. The difference with the original equations comes from the heat equation which now describes a simple balance between thermal diffusion and vertical advection against the mean temperature gradient.

We found that, in the asymptotic regime, (i) the processes of thermal diffusion $t_{\kappa}$ and of the restoring buoyancy force $t_{N}^{2}$ become a unique process with time scale $t_{N}^{2} / t_{\kappa}$, (ii) thermal diffusion always favour motions; this is because all the kinetic energy transformed into thermal energy by buoyancy work is dissipated. Then, thermal diffusivity can no longer increase the kinetic energy dissipation, (iii) strongly anisotropic motions, either horizontal or vertical ones, are less affected by buoyancy, (iv) numerical simulations are much faster since 

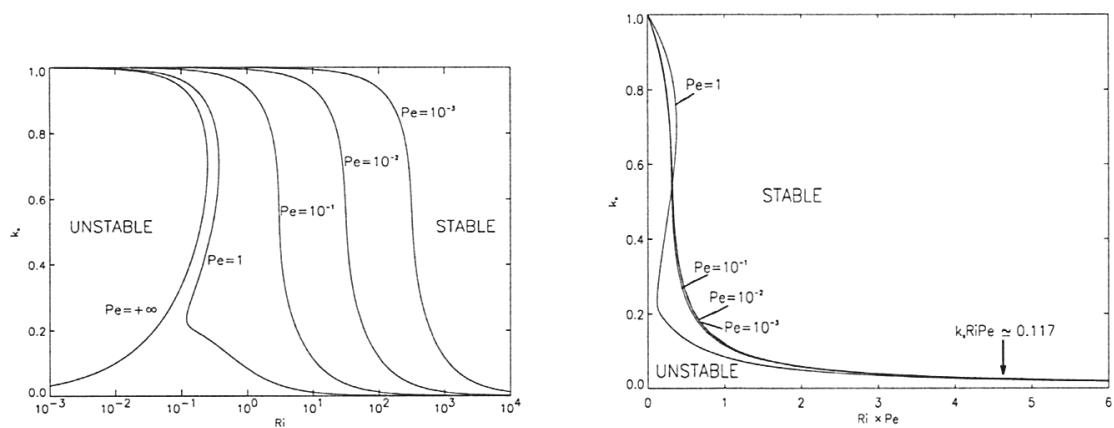

Figure 1. Curves of marginal stability of perturbations with horizontal wavenumber $k_{x}$, for different values of the Péclet number, as a function of $R i$ (left) and of $R=R i P e$ (right). The collapse of the $P e=10^{-2}$ and $P e=10^{-3}$ curves show that the asymptotic regime of small Péclet number has been reached.

the numerical time step is no longer constrained by the thermal diffusion time at the grid scale.

The small-Péclet-number approximation proved very useful to understand shear instabilities of Kelvin-Helmholtz type. We considered a parallel flow $\mathbf{U}=$ $\Delta U \tanh (z) \mathbf{e}_{x}$ in an unbounded vertically stratified Boussinesq atmosphere, the thermal stratification being linear. One of our objective was to test the criterion proposed by Zahn (1974) on phenomenological arguments. This criterion states that a shear is stable if, $R i P e_{t}>1 / 4$, where $P e_{t}=u l / \kappa$ is a Péclet number associated with an eddy of size $l$ and velocity $u$.

The main results of our analysis are that, (i) the small-Péclet-number regime is reached and for relatively large Péclet number $(P e \approx 0.1)$. Figure 1 shows evidences of this fact, (ii) the physical interpretation of the stability properties is greatly simplified in this regime since the effects of stable stratification and thermal diffusion combine in a single process, (iii) there exists two different types of unstable modes. The slowest growing modes correspond to predominantly horizontal motions and the fast growing ones to isotropic motions.

In conclusion, the effect of stable stratification and thermal diffusion on the dynamics is controlled by one relevant parameter in the limit of high thermal diffusion, that is, $R=R i P e$. This is fully consistent with Zahn (1974) stability criterion. Indirect evidences pointing towards small effective values of the Péclet number inside stellar radiative zones, we believe that the small-Pécletapproximation shall be useful to study turbulent transport in these regions. 3D numerical simulations are planned to test the prescriptions on the turbulent transport that are used in 1D or 2D stellar evolution codes.

\section{References}

Lignières, F. 1999, A\&A 348, 933

Lignières, F., Califano, F., Mangeney, A. 1999, A\&A 349, 1027

Zahn, J.P. 1974, in Stellar Instabilities and Evolution, IAU Symp. 59, p. 185 